九州大学学術情報リポジトリ

Kyushu University Institutional Repository

\title{
PROPERTIES OF PARTIALLY EFFICIENCY-BALANCED DESIGNS
}

\section{Kageyama, Sanpe i}

Department of Mathematics, Faculty of School Education, Hiroshima University

Puri, P. D.

Department of Mathematics and Statistics, Haryana Agricultural University

https://doi.org/10.5109/13365

出版情報: Bulletin of informatics and cybernetics. 21 (3/4), pp.19-28, 1985-03. Research Association of Statistical Sciences

バージョン :

権利関係 : 


\title{
PROPERTIES OF PARTIALLY EFFICIENCY- BALANCED DESIGNS
}

\author{
By \\ Sanpei Kageyama* and P. D. PURI**
}

\begin{abstract}
We consider a class of partially efficiency-balanced (PEB) designs which can be constructed in varying replicates and/or varying block sizes. These designs may be particularly useful for bio-assays, comparative varietal trials and factorial experiments. This paper gives some bounds on design parameters, some parametric relations, dual designs, complementary designs and some simple construction-methods for PEB designs.
\end{abstract}

\section{Introduction}

Block designs are widely used in many fields of research. A wide range of "Balanced" and "Partially Balanced" incomplete block designs are available in literature. However, most of the known designs are restricted to equal numbers of replications and block sizes. The practical considerations often dictate the use of varying replicate and varying block-sized designs. We shall here consider a class of incomplete block designs called "Partially Efficiency balanced designs" introduced by Puri and Nigam [6]. These designs are available in varying replicates and/or varying block sizes, and thus give experimenters more freedom in designing experiments in unconventional circumstances. These designs have simple analysis and also allow the important contrasts to be estimated and tested with desired efficiency.

Consider a block design $D(v, b, \boldsymbol{r}, \boldsymbol{k})$ with $v$ treatments arranged in $b$ blocks such that the $i$-th treatment is replicated $r_{i}$ times $(i=1,2, \cdots, v)$ and $j$-th block is of size $k_{j}(j=1,2, \cdots, b)$ with $\boldsymbol{r}^{\prime}=\left(r_{1}, r_{2}, \cdots, r_{v}\right)$ and $\boldsymbol{k}^{\prime}=\left(k_{1}, k_{2}, \cdots, k_{b}\right)$. In particular, if $r_{i}=r$ for all $i$, the design is said to be equireplicated and it is said to be equiblock-sized if $k_{j}=k$ for all $j$.

Let $N=\left(\left(n_{i j}\right)\right)$ be the $v \times b$ incidence matrix, where $n_{i j}$ denotes the number of times $i$-th treatment occurs in $j$-th block. When $n_{i j}=0$ or 1 for all $i, j$, the design is said to be binary, and it is said to be $p$-ary if $n_{i j}$ takes $p$ distinct integral values.

For the analysis of block designs in general Tocher [10] has defined $\Omega^{-1}$-matrix as $\Omega^{-1}=R-N K^{-1} N^{\prime}+(1 / n) \boldsymbol{r} \boldsymbol{r}^{\prime}$ where $R$ and $K$ are diagonal matrices with diagonal ele-

* Department of Mathematics, Faculty of School Education, Hiroshima Lniversity, Shinonome, Hiroshima 734, Japan.

** Department of Mathematics and Statistics, Haryana Agricultural Lniversity, Hissar 125004, India. 
ments $r_{1}, r_{2}, \cdots, r_{n}$ and $k_{1}, k_{2}, \cdots, k_{b}$, respectively, and $n$ denotes the total number of experimental units (i. e., $n=\sum_{i=1}^{D} r_{i}=\sum_{j=1}^{b} k_{j}$ ). The main task of analysis of design is to obtain the inverse of $\Omega^{-1}$-matrix. An iterative method of inverting $\Omega^{-1}$-matrix is given by Caliński $[1]$ in terms of eigenvalues of "Information matrix"

$$
M_{0}=M-(1 / n) \mathbf{1} \boldsymbol{r}^{\prime}
$$

where $M=R^{-1} N K^{-1} N^{\prime}$. Here 1 is a column vector of units.

A design $D(v, b, \boldsymbol{r}, \boldsymbol{k})$ is called a partially efficiency-balanced (PEB) design with $m$ efficiency-classes if there exists a set of $v-1$ mutually orthogonal contrasts $\left\{\boldsymbol{s}_{i j}\right\}$ which can be partitioned into $m$ disjoint classes such that all the $\alpha_{i}$ contrasts of $i$-th class are estimated with relative loss of information $\mu_{i}$, i. e., they satisfy

$$
M_{0} \boldsymbol{s}_{i j}=\mu_{i} \boldsymbol{s}_{i j} ; i=1,2, \cdots, m ; j=1,2, \cdots, \alpha_{i} .
$$

The parameters of a PEB design can be written as $v, b, \boldsymbol{r}, \boldsymbol{k}, \mu_{i}, \alpha_{i}, L_{i} ; i=1,2, \cdots$, $m$. It is clear from the definition that a PEB design can be characterized as an incidence matrix $N$, satisfying the spectral expansion

$$
M\left(=R^{-1} N K^{-1} N^{\prime}\right)=\sum_{i=0}^{m} \mu_{i} L_{i}
$$

or

$$
M_{0}\left(=M-(1 / n) \mathbf{1} \boldsymbol{r}^{\prime}\right)=\sum_{i=1}^{m} \mu_{i} L_{i}
$$

such that $\sum_{i=0}^{m} L_{i}=I$ where $\mu_{0}=1$ and $L_{0}=(1 / n) 1 \boldsymbol{r}^{\prime}$. Here $I$ is the identity matrix of appropriate order. For a PEB design, the $\Omega$-matrix takes the simplified form

$$
\Omega=\left[I+\sum_{i=1}^{m}\left\{\mu_{i} /\left(1-\mu_{i}\right)\right\} L_{i}\right] R^{-1} .
$$

A particular class of PEB designs where $\mu_{i}$ takes only two distinct values, $\mu_{1} \neq 0$ and $\mu_{2}=0$, with multiplicities $\alpha_{1}$ and $\alpha_{2}\left(=v-\alpha_{1}-1\right)$, respectively, is of special interest, as a set of $\alpha_{2}(>0)$ contrasts is estimated with full efficiency. Such a class of designs has very simple analysis and is termed as simple (S-PEB) designs by Puri and Nigam [6]. If $\mu_{i}=\mu$ for all $i$, the design is called an Efficiency Balanced (EB) design. For such a design, $M_{0}$ has only one non-zero eigenvalue $\mu$ with multiplicity $v-1$, and every contrast is estimated with the same efficiency.

Since a design uniquely determines its incidence matrix and conversely, both the design and its incidence matrix are denoted by the same symbol throughout this paper.

In this paper, we shall investigate various fundamental properties of PEB designs. Some bounds on parameters and some parametric relations of PEB designs are given. Dual design, complementary design and some simple methods of construction are also presented. 


\section{Eigenvalues of Information Matrix}

Efficiency-factors $1-\mu_{i}$ associated with the $\alpha_{i}$ contrasts of $i$-th class are very important for PEB designs. In this section, we shall investigate some behavior about the eigenvalues $\mu_{i}$ of the matrix $M$. At first we have the following.

PROPOSITION 2.1. For a connected PEB design, the maximum eigenvalue $\mu_{0}=1$ is simple, and other eigenvalues $\mu_{i}$ satisfy $0 \leqq \mu_{i}<1$ for $i=1,2, \cdots, m$.

Proof. It is well-known (cf. Yamamoto and Fujikoshi [12]) that the eigenvalues $\theta_{i}$ of $R^{-1}\left(R-N K^{-1} N^{\prime}\right)$ satisfy $0 \leqq \theta_{i} \leqq 1$, and, for a connected design, the minimum eigenvalue, 0 , is simple. Now, it follows from the definition of a PEB design that

$$
R^{-1}\left(R-N K^{-1} N^{\prime}\right)=\sum_{i=0}^{m}\left(1-\mu_{i}\right) L_{i}
$$

which, from the above observation with $\theta_{i}=1-\mu_{i}$, completes the proof.

The original definition of a PEB design is not so clear about statements of eigenvalues $\mu_{i}$. From Proposition 2.1, we can state that in the expression of $M=\sum_{i=0}^{m} \mu_{i} L_{i}$ for a connected PEB design,

(i) the figure " $m$ " (in $m$ efficiency-classes) is equal to the number of distinct eigenvalues of $M_{0}$;

(ii) $\mu_{0}=1$ with multiplicity 1 ;

(iii) for other eigenvalus for $i \geqq 1,0 \leqq \mu_{i}<1$.

We deal only with connected designs throughout this paper. Next, we shall evaluate extreme eigenvalues, i. e., $\mu_{\max }=\max \left\{\mu_{1}, \mu_{2}, \cdots, \mu_{m}\right\}$ and $\mu_{\min }=\min \left\{\mu_{1}, \mu_{2}, \cdots, \mu_{m}\right\}$. But, $\mu_{\min }$ may be zero for PEB designs. If $\mu_{\min }$ is not zero, we can evaluate it too.

PROPOSITION 2.2. For a PEB design with $\mu_{\min } \neq 0$,

$$
\mu_{\max } \geqq\left[\operatorname{tr}\left(R^{-1} N K^{-1} N^{\prime}\right)-1\right] /(v-1) \geqq \mu_{\min },
$$

both equalities holding if and only if $\mu_{1}=\mu_{2}=\cdots=\mu_{m}$ (i.e., the design is an EB design).

PROOF. From the definition of a PEB design, we have

$$
R^{-1} N K^{-1} N^{\prime}-(1 / n) \mathbf{1} \boldsymbol{r}^{\prime}=\sum_{i=1}^{m} \mu_{i} L_{i}
$$

where $\operatorname{rank}\left(L_{i}\right)=\alpha_{i}, i=1,2, \cdots, m$. By taking the trace of the both sides of $(2.2)$, it holds that

$$
\begin{aligned}
\operatorname{tr}\left(R^{-1} N K^{-1} N^{\prime}\right)-1 & =\sum_{i=1}^{m} \mu_{i} \alpha_{i} \\
& \leqq(v-1) \mu_{\max },
\end{aligned}
$$

since $\sum_{i=1}^{m} \alpha_{i}=v-1$. On the other hand, since $\mu_{\min } \neq 0$, (2.3) yields the required bound on $\mu_{\min }$. This completes the proof.

When the design is binary, we have the following.

COROLLARY 2.2.1. For a binary PEB design with $\mu_{\min } \neq 0$,

$$
\mu_{\max } \geqq\left[\sum_{i=1}^{v} \frac{1}{r_{i}}\left(\sum_{j=1}^{b} \frac{n_{i j}}{k_{j}}\right)-1\right] /(v-1) \geqq \mu_{\min },
$$


both equalities holding if and only if non-zero $\mu_{i}$ 's are constant.

COROLlary 2.2.2. For an equireplicated binary PEB design with $\mu_{\min } \neq 0$,

$$
\mu_{\max } \geqq(b-r) /[r(v-1)] \geqq \mu_{\min } .
$$

COROLlary 2.2.3. For an equiblock-sized binary PEB design with $\mu_{\min } \neq 0$,

$$
\mu_{\max } \geqq(v-k) /[k(v-1)] \geqq \mu_{\min } .
$$

REMARK. When the design is equireplicated and equiblock-sized, $(b-r) /[r(v-1)]=$ $(v-k) /[k(v-1)]$.

For a PEB design with parameters $v, b, \boldsymbol{r}, \boldsymbol{k}, \mu_{i}, \alpha_{i}, L_{i}, i=1,2, \cdots, m$, the eigenvalues $\mu_{i}$ 's are loss of information associated with the $\alpha_{i}$ contrasts of $i$-th class. In this sense, we can call $\sum_{i=1}^{m} \mu_{i} \alpha_{i}$ to be the total loss of information for this design, where $\alpha_{i}=\operatorname{rank}\left(L_{i}\right)$. From the information matrix, as in (2.3)

$$
\sum_{i=1}^{m} \mu_{i} \alpha_{i}=\operatorname{tr}\left(R^{-1} N K^{-1} N^{\prime}\right)-1
$$

In particular, if the design is binary, then

$$
\sum_{i=1}^{m} \mu_{i} \alpha_{i}=\sum_{i=1}^{v} \frac{1}{r_{i}} \sum_{j=1}^{b} \frac{n_{i j}}{k_{j}}-1
$$

In this case, we can present a bound on the total loss of information.

Proposition 2.3. For a binary PEB design,

$$
\frac{v}{\max k_{j}}-1 \leqq \sum_{i=1}^{m} \mu_{i} \alpha_{i} \leqq \frac{v}{\min k_{j}}-1,
$$

where $\max k_{j}=\max \left\{k_{1}, k_{2}, \cdots, k_{b}\right\}$ and $\min k_{j}=\min \left\{k_{1}, k_{2}, \cdots, k_{b}\right\}$.

PROOF. It generally holds that for a block design

$$
r_{i} /\left(\max k_{j}\right) \leqq \sum_{j=1}^{b} n_{i j} / k_{j} \leqq r_{i} /\left(\min k_{j}\right) \quad \text { for all } i
$$

which yield

$$
\frac{v}{\max k_{j}} \leqq \sum_{i=1}^{v} \frac{1}{r_{i}} \sum_{j=1}^{b} \frac{n_{i j}}{k_{j}} \leqq \frac{v}{\min k_{j}} .
$$

Since the design is binary, (2.4) and (2.5) complete the proof.

As special cases of Proposition 2.3, we have the following.

COROLLARY 2.3.1. For an equireplicated binary PEB design, the total loss of information is $b / r-1$.

COROLLARY 2.3.2. For an equiblock-sized binary PEB design, the total loss of information is $v / k-1$.

REMARK. Jones [2] derived essentially the same results as Corollaries 2.3.1 and 2.3.2 for equireplicated and equiblock-sized designs.

As a special type of PEB designs, we now consider an S-PEB design which has the only one non-zero eigenvalue $\mu$ with multiplicity $\alpha_{1}$ for the information matrix. In this case we have the following.

Proposition 2.4. For an S-PEB design with $v \geqq 3$, 


$$
\left[\operatorname{tr}\left(R^{-1} N K^{-1} N^{\prime}\right)-1\right] /(v-1) \leqq \mu \leqq\left[\operatorname{tr}\left(R^{-1} N K^{-1} N^{\prime}\right)-1\right] / 2,
$$

(i) the equality at the left-hand side holding if and only if $\alpha_{1}=v-1$ (i.e., the design is an EB design), and (ii) the equality of the right-hand side holding if and only if $\alpha_{1}=2$.

Proof. For an S-PEB design with $1>\mu>0$, (2.3) yields $\operatorname{tr}\left(R^{-1} N K^{-1} N^{\prime}\right)-1=\mu \alpha_{1}$, where $\alpha_{1}=\operatorname{rank}\left(L_{1}\right)$ and, in general, $1 \leqq \alpha_{1} \leqq v-1$. When $\alpha_{1}=v-1$, it follows that the S-PEB design becomes an EB design. When $\alpha_{1}=1$, it follows from (2.1) that for an S-PEB design

$$
\operatorname{rank}\left(R-N K^{-1} N^{\prime}\right)=\operatorname{rank}\left[(1-\mu) L_{1}\right]=\operatorname{rank}\left(L_{1}\right)=1 .
$$

Since the design is connected, $\operatorname{rank}\left(R-N K^{-1} N^{\prime}\right)=v-1$ which, from (2.6), yields $v=2$. This is a contradiction to an assumption of $v \geqq 3$. Hence, $\alpha_{1} \geqq 2$. This completes the proof.

REMARK. When $v=3$, Proposition 2.4 gives an exact expression on $\mu$.

For binary designs, this proposition yields the following.

COROLlary 2.4.1. For a binary $S-P E B$ design with $v \geqq 3$,

$$
\left[\sum_{i=1}^{v} \frac{1}{r_{i}} \sum_{j=1}^{b} \frac{n_{i j}}{k_{j}}-1\right] /(v-1) \leqq \mu \leqq\left[\sum_{i=1}^{v} \frac{1}{r_{i}} \sum_{j=1}^{b} \frac{n_{i j}}{k_{j}}-1\right] / 2 .
$$

COROLLARY 2.4.2. For an equireplicated binary $S-P E B$ design with $v \geqq 3$,

$$
(b-r) /[r(v-1)] \leqq \mu \leqq(b / r-1) / 2 .
$$

COROLLARY 2.4.3. For an equiblock-sized binary S-PEB design with $v \geqq 3,(v-k) /$ $[k(v-1)] \leqq \mu \leqq(v / k-1) / 2$.

REMARK. When the design is equireplicated and equiblock-sized, Corollaries 2.4.2 and 2.4.3 are the same.

\section{Bounds on the Number of Blocks}

Some discussions about the expression of lower bounds on the number of blocks for a block design are available in literature. Similarly, we can produce a lower bound on the number of blocks for a PEB design by following Kageyama's approach [3].

Proposition 3.1. For a PEB design, $b \geqq v-\delta$ holds, where $\delta$ is the multiplicity of zero eigenvalue of the $M$-matrix. In particular, the equality sign holds if and only if the projection corresponding to zero eigenvalue of $K^{-1} N^{\prime} R^{-1} N$ is a zero matrix. In this case,

$$
K=N^{\prime}\left[(1 / n) \mathbf{1} \boldsymbol{r}^{\prime}+\sum_{i}\left(1 / \mu_{i}\right) L_{i}\right] R^{-1} N
$$

where the summation extends over all the integers $i$ satisfying $\mu_{i}>0$ for $i=1,2, \cdots, m$.

PROOF. Let $\delta$ be the multiplicity of zero eigenvalue of the relation (1.1), which yields

$$
v-\delta=\operatorname{rank}\left(R^{-1} N K^{-1} N^{\prime}\right)=\operatorname{rank}\left(N K^{-1} N^{\prime}\right)=\operatorname{rank}(N) \leqq b,
$$

i. e., an inequality $b \geqq v-\delta$ holds. Furthermore, from (1.1) we have a spectral expansion of $K^{-1} N^{\prime} R^{-1} N$ as

$$
K^{-1} N^{\prime} R^{-1} N=K^{-1} N^{\prime} L_{0} R^{-1} N+\sum_{2} \mu_{i}\left[\left(1 / \mu_{i}\right) K^{-1} N^{\prime} L_{i} R^{-1} N\right]+0 \cdot Q,
$$


where $Q$ is the projection corresponding to zero with $\operatorname{rank}(Q)=b-(v-\delta)$ and the summation $\sum_{i}$ extends over all the integers $i$ satisfying $\mu_{i}>0$ for $i=1,2, \cdots, m$. Hence it follows that $b=v-\delta$ if and only if $Q$ is a zero matrix, in which case

$$
I_{b}=K^{-1} N^{\prime} L_{0} R^{-1} N+\sum_{i}\left(1 / \mu_{i}\right) K^{-1} N^{\prime} L_{i} R^{-1} N .
$$

Thus, the proof is completed.

Letting $\delta=0$ in Proposition 3.1, we have the following.

COROLLARY 3.1.1. For a PEB design, if $\mu_{i}>0$ for all $i=1,2, \cdots, m$, then the Fisher inequality $b \geqq v$ holds.

An equireplicated block design is said to be $\alpha$-resolvable if the blocks can be separated into $t$ sets of $\beta$ blocks each $(b=\beta t)$ such that each set contains every treatment exactly $\alpha$ times $(r=\alpha t)$. In this case, the following is obtained.

Proposition 3.2. For an $\alpha$-resolvable PEB design with $b=\beta$ t and $r=\alpha t, b \geqq v+t-$ $\delta-1$ holds, where $\delta$ is the multiplicity of zero eigenvalue of the M-matrix.

PROOF. From the definition of $\alpha$-resolvability, the sum of the columns corresponding to each set in the incidence matrix $N$ must give a column consisting of $\alpha$ 's. Hence, not more than $b-t+1$ column vectors are independent. Thus, $\operatorname{rank}(N) \leqq b-t+1$, which, from (3.1), yields the required result.

\section{Dual of PEB Designs}

We here consider a dual of a PEB design $N$ with parameters $v, b, \boldsymbol{r}, \boldsymbol{k}, \mu_{i}, L_{i}, i$ $=0,1, \cdots, m$, having $\mu_{0}=1$ and $L_{0}=(1 / n) 1 \boldsymbol{r}^{\prime}$. In this case, we have the relation (1.1). As an incidence matrix of a dual design, we can utilize $N^{\prime}$, whose $M$-matrix is given by

$$
M^{*}=K^{-1} N^{\prime} R^{-1} N=\left(K^{-1} N^{\prime}\right)\left(R^{-1} N\right) .
$$

In this case it follows from (1.1) that

$$
\begin{aligned}
M^{*} & =K^{-1} N^{\prime} L_{0} R^{-1} N+\sum_{i} \mu_{i}\left[\left(1 / \mu_{i}\right) K^{-1} N^{\prime} L_{i} R^{-1} N\right]+0 \cdot Q \\
& =(1 / n) 1 k^{\prime}+\sum_{i} \mu_{i}\left[\left(1 / \mu_{i}\right) K^{-1} N^{\prime} L_{i} R^{-1} N\right] \\
& =L_{0}^{*}+\sum_{i} \mu_{i} L_{i}^{*},
\end{aligned}
$$

where $Q$ is the projection corresponding to zero eigenvalue of $M^{*}$, the summation extends over all the integers $i$ satisfying $\mu_{i}>0$ for $i=1,2, \cdots, m$, and for such $i L_{i}^{*}=$ $\left(1 / \mu_{i}\right) K^{-1} N^{\prime} L_{i} R^{-1} N, L_{0}^{*}=(1 / n) \mathbf{1} \boldsymbol{k}^{\prime}, Q=I_{b}-L_{0}^{*}-\sum_{i} L_{i}^{*}$. Hence the dual design has at most $m+1$ efficiency-classes.

Thus, we have established the following.

Proposition 4.1. The dual of a PEB design with parameters $v, b, \boldsymbol{r}, \boldsymbol{k}, \mu_{i}, L_{i}, i=0$, $1, \cdots, m$, is a PEB design, having at most $m+1$ efficiency-classes, with parameters $v^{*}=b$, $b^{*}=v, \boldsymbol{r}^{*}=\boldsymbol{k}, \boldsymbol{k}^{*}=\boldsymbol{r}, \mu_{0}=1, \mu_{i}, L_{i}^{*}$ such that $L_{0}^{*}=(1 / n) \mathbf{1} \boldsymbol{k}^{\prime}, L_{i}^{*}=\left(1 / \mu_{i}\right) K^{-1} N^{\prime} L_{i} R^{-1} N, Q=$ $I_{b}-L_{0}^{*}-\sum_{i} L_{i}^{*}$, the summation extending over all the integers $i$ satisfying $\mu_{i}>0$ for $i=$ $1,2, \cdots, m$. 
REMARK. If all $\mu_{i}$ 's are positive for $i=1,2, \cdots, m$, then the dual design has "exactly" $m+1$ efficiency-classes. Singh [9] considered only this case for the dual of a PEB design. In this sense, Singh's result is a special case of Proposition 4.1.

Nigam and Puri [5] considered the dual of S-PEB and symmetrical PEB designs which are both equireplicated and equiblock-sized, under some restriction of inner structure of the incidence matrix. Thus their results are also particular cases of Proposition 4.1 .

The observation in Proposition 4.1 is useful to construct some types of PEB designs.

\section{Some Properties}

We here present some other properties and observations on simple constructions. From the definition of a PEB design, we have $R^{-1} N K^{-1} N^{\prime}=\sum_{i=0}^{m} \mu_{i} L_{i}$ whose determinant is

$$
\left|R^{-1} N K^{-1} N^{\prime}\right|=\mu_{1}^{\alpha} \mu_{2}^{\alpha_{2}} \cdots \mu_{m}^{\alpha} m .
$$

In this case, immediately we can obtain the following.

Proposition 5.1. For a PEB design, if $v>b$, then some eigenvalues $\mu_{i}$ must be zero.

REMARK. Proposition 5.1 also yields Corollary 3.1.1, and further suggests that there may be the possibility for a PEB design with $b \geqq v$ to have more many efficiency-classes than a PEB design with $b<v$.

Proposition 5.2. For a PEB design, if $v=b$, then $\prod_{i=1}^{v} r_{i} k_{i} \prod_{j=1}^{m} \mu_{j}^{\alpha_{j}}$ is a perfect square.

Proof. When $v=b,(5.1)$ yields

$$
|N|^{2}=r_{1} r_{2} \cdots r_{v} k_{1} k_{2} \cdots k_{v} \mu_{1}^{\alpha_{1}} \mu_{2}^{\alpha} \cdots \mu_{m}^{\alpha} m
$$

which completes the proof.

The $C\left(=R-N K^{-1} N^{\prime}\right)$-matrix plays an important role in a block design. In this case, we have the following relation.

Proposition 5.3. For a PEB design $C=R\left[\sum_{i=1}^{m}\left(1-\mu_{i}\right) L_{i}\right]$.

Proof is obvious from (2.1).

REMARK. An equireplicated PEB design has a fine structure of the $C$-matrix.

When $m=1$ and $\mu_{1} \neq 0$, Proposition 5.3 yields a more practical result.

COROLlARY 5.3.1. For an $E B$ design $C=\left(1-\mu_{1}\right)\left[R-(1 / n) \boldsymbol{r} \boldsymbol{r}^{\prime}\right]$.

REMARK. Corollary 5.3.1 is the same result as in Kageyama [4].

Now, we may define the complement of a $p$-ary design whose incidence matrix is $N=\left(\left(n_{i j}\right)\right)$ with $n_{i j}=0,1, \cdots$, or $p-1$, as $\overline{\boldsymbol{N}}=\left(\left(\overline{\boldsymbol{n}}_{i j}\right)\right)$ with $\overline{\boldsymbol{n}}_{i j}=(p-1)-n_{i j}$ for all $i, j$. In this case, we have the following.

PROPOSITION 5.4. The complement of a p-ary PEB design with parameters $v, b, r, k$, $\mu_{i}, L_{i}, i=1,2, \cdots, m$, is a p-ary PEB design with parameters $v^{\prime}=v, b^{\prime}=b, r^{\prime}=(p-1) b-r$, $k^{\prime}=(p-1) v-k, \mu_{i}^{\prime}=r k \mu_{i} /[\{(p-1) b-r\}\{(p-1) v-k\}], L_{i}, i=1,2, \cdots, m$.

Proof. From the definition,

$$
N N^{\prime}=r k \sum_{i=0}^{m} \mu_{i} L_{i}
$$


Since $\bar{N}=(p-1) J_{v \times b}-N$ where $J_{v \times b}=1_{v} 1_{b}^{\prime}$, it holds that

$$
\bar{N} \mathbf{1}=[(p-1) b-r] \mathbf{1}, \mathbf{1}^{\prime} \overline{\mathbf{N}}=[(p-1) v-k] \mathbf{1}^{\prime}
$$

which imply that $r^{\prime}=(p-1) b-r$ and $k^{\prime}=(p-1) v-k$. Furthermore, it follows from (5.2) that the information matrix of the complementary design can be given by $\sum_{i=1}^{m} \mu_{i}^{\prime} L_{i}$, where $\mu_{i}^{\prime}=r k \mu_{i} /[\{(p-1) b-r\}\{(p-1) v-k\}]$ for $i \geqq 1$. Thus, the proof is completed.

Note that $\mu_{i}^{\prime}<\mu_{i} \Leftrightarrow b>2 r /(p-1)$. This means that when $b>2 r /(p-1)$, the complementary design is more efficient than the original design. When $p=2$, Proposition 5.4 yields the following.

COROLlary 5.4.1. The complement of a binary PEB design with parameters $v, b, r$, $k, \mu_{i}, L_{i}, i=1,2, \cdots, m$, is a binary $P E B$ design with parameters $v^{\prime}=v, b^{\prime}=b, r^{\prime}=b-r$, $k^{\prime}=v-k, \mu_{i}^{\prime}=r k \mu_{i} /[(b-r)(v-k)], L_{i}, i=1,2, \cdots, m$.

We will present a number of methods of constructions for PEB designs in other subsequent papers. However we here state some new fundamental methods of constructions of PEB designs. For some available construction-methods, refer to Puri and Nigam [6], Puri, et al. [7] and Nigam and Puri [5].

Let $N_{l}$ be two PEB designs with parameters $v, b_{l}, \boldsymbol{r}_{l}, \boldsymbol{k}_{l}, \mu_{i}^{(l)}, L_{i}, i=0,1, \cdots, m$ for $l=1,2$. Then we consider the juxtaposition of these designs, $\left[N_{1}: N_{2}\right]$, whose $M$-matrix can be given by

$$
M^{*}=\left(R_{1}+R_{2}\right)^{-1} \sum_{i=0}^{m}\left(\mu_{i}^{(1)} R_{1}+\mu_{i}^{(2)} R_{2}\right) L_{i}
$$

where $R_{l} \mathbf{1}=\boldsymbol{r}_{l}, l=1,2$. In this case, we have the following.

Proposition 5.5. When $N_{l}$ are PEB designs with parameters $v, b_{l}, \boldsymbol{r}, \boldsymbol{k}_{l}, \mu_{i}^{(l)}, L_{i}$, $i=1,2, \cdots, m$ and $l=1,2,\left[N_{1}: N_{2}\right]$ is a PEB design with parameters $v^{*}=v, b^{*}=b_{1}+b_{2}$, $\boldsymbol{r}^{*}=2 \boldsymbol{r}, \boldsymbol{k}^{* \prime}=\left[\boldsymbol{k}_{1}^{\prime}, \boldsymbol{k}_{2}^{\prime}\right], \mu_{i}^{*}=\left(\mu_{i}^{(1)}+\mu_{i}^{(2)}\right) / 2, L_{i}^{*}=L_{i}, i=1,2, \cdots, m$.

Proof. When $\boldsymbol{r}_{1}=\boldsymbol{r}_{2}=\boldsymbol{r}$, (5.3) yields $M^{*}=\sum_{i=0}^{m}\left[\left(\mu_{i}^{(1)}+\mu_{i}^{(2)}\right) / 2\right] L_{i}$ which yields the required result.

Proposition 5.6. When $N_{l}$ are PEB designs with parameters $v, b_{l}, \boldsymbol{r}_{l}=r_{l} \mathbf{1}, \boldsymbol{k}_{l}, \mu_{i}^{(l)}$, $L_{i}$ for $i=1,2, \cdots, m$ and $l=1,2,\left[N_{1}: N_{2}\right]$ is a PEB design with parameters $v^{*}=v, b^{*}$ $=b_{1}+b_{2}, \boldsymbol{r}^{*}=\left(r_{1}+r_{2}\right) \mathbf{1}, \boldsymbol{k}^{* \prime}=\left[\boldsymbol{k}_{1}^{\prime}, \boldsymbol{k}_{2}^{\prime}\right], \mu_{i}^{*}=\left(r_{1} \mu_{i}^{(1)}+r_{2} \mu_{i}^{(2)}\right) /\left(r_{1}+r_{2}\right), L_{i}^{*}=L_{i}, i=1,2, \cdots, m$.

PROOF. When $\boldsymbol{r}_{l}=r_{l} \mathbf{1}$ for $l=1,2$, (5.3) yields

$$
M^{*}=\sum_{i=0}^{m}\left[\left(r_{1} \mu_{i}^{(1)}+r_{2} \mu_{i}^{(2)}\right) /\left(r_{1}+r_{2}\right)\right] L_{i}
$$

which yields the proposition.

REMARK. In Propositions 5.5 and 5.6, $\mu_{i}^{*}<\mu_{i}^{(1)} \Leftrightarrow \mu_{i}^{(2)}<\mu_{i}^{(1)}$, and $\mu_{i}^{*}<\mu_{i}^{(2)} \Leftrightarrow \mu_{i}^{(1)}<\mu_{i}^{(2)}$.

The methods in Propositions 5.5 and 5.6 provide practical criterions for the juxtaposition of PEB designs to become a PEB design, and can easily be generalized to a case of the juxtaposition of a finite number of PEB designs. As special cases of Proposition 5.6, we have the following.

COROllary 5.6.1. When $N$ is a PEB design with parameters $v, b, r, \boldsymbol{k}, \mu_{i}, L_{i}, i=$ $1,2, \cdots, m,\left[N: I_{v}\right]$ is a PEB design with parameters $v^{*}=v, b^{*}=b+v, r^{*}=r+1, \boldsymbol{k}^{* \prime}=$ $\left[\boldsymbol{k}^{\prime}, \mathbf{1}^{\prime}\right], \mu_{i}^{*}=\left(r \mu_{i}+1\right) /(r+1), i=1,2, \cdots, m$. 
REMARK. In Corollary 5.6.1, $\mu_{i}^{*}>\mu_{i}$ for $i \geqq 1$.

COROllary 5.6.2. When $N$ is a PEB design with parameters $v, b, r, \boldsymbol{k}, \mu_{i}, L_{i}, i=$ $1,2, \cdots, m,\left[N: J_{v \times l}\right]$ is a PEB design with parameters $v^{*}=v, b^{*}=b+l, r^{*}=r+l, k^{* \prime}=$ $\left[\boldsymbol{k}^{\prime}, v \mathbf{1}^{\prime}\right], \mu_{i}^{*}=r \mu_{i} /(r+l), L_{i}, i=1,2, \cdots, m$ and $l \geqq 1$.

REMARK. In Corollary 5.6.2, $\mu_{i}^{*}<\mu_{i}$ for $i \geqq 1$, and so the resultant design may always be more efficient. Corollaries 5.6.1 and 5.6.2 suggest that PEB designs having more large numbers of experimental units may be more efficient.

COROLlaRy 5.6.3. When $N$ is a PEB design with parameters $v, b, r, \boldsymbol{k}, \mu_{i}, L_{i}, i=$ $1,2, \cdots, m,[N: J-I]$ is a PEB design with paraneters $v^{*}=v, b^{*}=b+v, r^{*}=r+v-1, \boldsymbol{k}^{* \prime}$ $=\left[\boldsymbol{k}^{\prime},(v-1) \mathbf{1}^{\prime}\right], \mu_{i}^{*}=\left[(v-1) r \mu_{i}+1\right] /[(v-1)(r+v-1)], L_{i}, i=1,2, \cdots, m$.

REMARK. In Corollary 5.6.3, $\mu_{i}^{*}<\mu_{i} \Leftrightarrow \mu_{i}>1 /(v-1)^{2}$ for $i \geqq 1$.

From Corollary 5.4.1 and Proposition 5.6, we have the following.

COROLlaRy 5.6.4. When $N$ is a binary PEB design with parameters $v, b, r, k, \mu_{i}$, $L_{i}, i=1,2, \cdots, m,[N: \bar{N}]$ is a PEB design with parameters $v^{*}=v, b^{*}=2 b, r^{*}=b, \boldsymbol{k}^{* \prime}=$ $\left[k \mathbf{1}^{\prime},(v-k) \mathbf{1}^{\prime}\right], \mu_{i}^{*}=k \mu_{i} /(v-k), L_{i}, i=1,2, \cdots, m$.

REMARK. In Corollary 5.6.4, $\mu_{i}^{*}<\mu_{i} \Leftrightarrow v>2 k$. Furthermore, Corollaries 5.4.1 and 5.6.4 imply that for an equireplicated, equiblock-sized PEB design $N$ with $v>2 k, \bar{N}$ or $[N: \bar{N}]$ should be utilized as experimental plans, provided such practical change is allowed.

Since the dual of a PEB design is a PEB design (see Proposition 4.1), by taking the dual of the designs given in Propositions 5.5 and 5.6, and Corollaries 5.6.1 to 5.6.4, we can produce supplemented PEB designs. These discussions about constructions will be given in other papers.

Finally, we can draw the relationship amomg "notions" of some block designs.

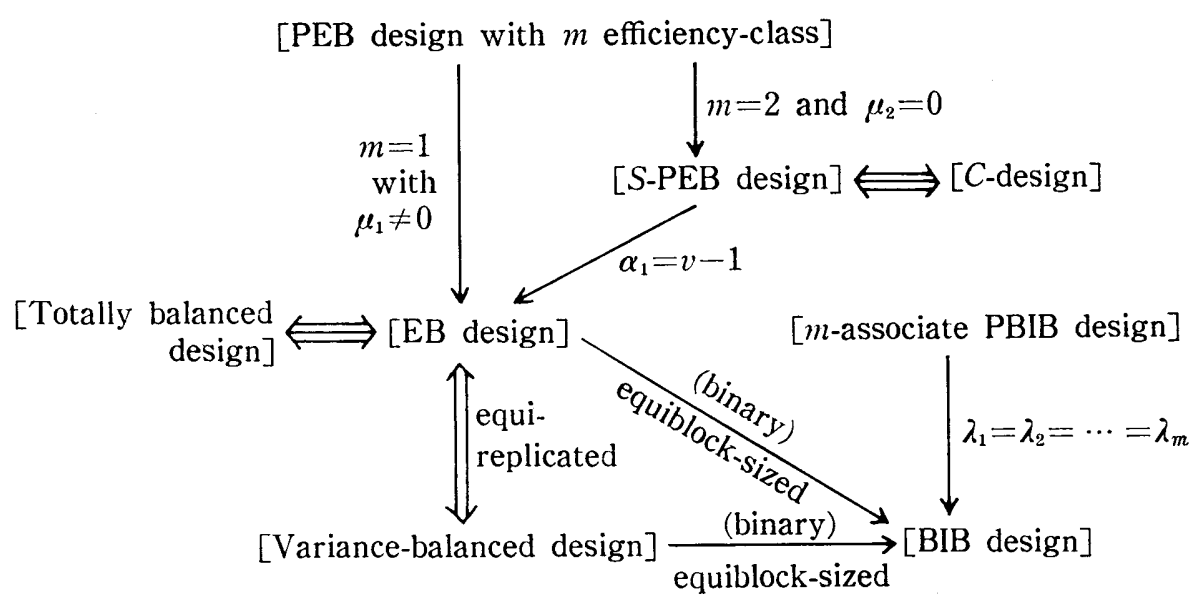

Symbol " $A \stackrel{c}{\rightarrow} B$ " means that $A$ implies $B$ under $C$; “ $A \Leftrightarrow B$ " means that $A$ is equivalent to $B$; “ $A \stackrel{c}{\Leftrightarrow} B$ " means that $A$ and $B$ are equivalent under $C$.

REMARK. $C$-designs are named by Saha [8], and totally balanced designs are first considered by Jones [2] and Caliński [1]. (i) A partially balanced incomplete block 
(PBIB) design is an equireplicated, equiblock-sized binary PEB design (cf. Puri and Nigam [6]). (ii) A supplemented block design, (partially) balanced factorial experiment, linked block design, and a block design having a general balance property of Wilkinson [11] are special cases of PEB designs. (iii) It is clear from the definition of a PEB design that any connected block design is a special case of a PEB design with at most $v-1$ efficiency-classes, where $v$ is the number of treatments. In this sense, a PEB design is so much general.

\section{References}

[1] Calí́ski, T.: On some desirable patterns in block designs. Biometrics, 27 (1971), 275-292.

[2] Jones, R.M.: On a property of incomplete blocks. J. Roy. Statist. Soc., B 21 (1959), 172179 .

[3] Kageyama, S.: Mathematical expression of an inequality for a block design. Ann. Inst. Statist. Math., 31 A (1979), 293-298.

[4] Kageyama, S.: On properties of efficiency-balanced designs. Commun. Statist. Theor. Meth., A 9 (1980), 597-616.

[5] Nigam, A.K. and PuRI, P.D. : On partially efficiency-balanced design-II. Commun. Statist. Theor. Meth., A 11 (1982), 2817-2830.

[6] Puri, P.D. and Nigam, A.K.: Partially efficiency balanced designs. Commun. Statist. Theor. Meth., A 6 (1977), 753-771.

[7] Puri, P.D., Nigam, A.K. and Narain, P.: Supplemented block designs. Sankhyā, B 39 (1977), 189-195.

[8] SAhA, G. M. : On Calinski's patterns in block designs. Sankhyā, B 38 (1976), 383-392.

[9] SingH, M.: Some constructions to design and analysis of balanced and partially balanced experiments. Unpublished Ph. D. Thesis : Institute of Agricultural Research Statistics, New Delhi, 1976.

[10] Tocher, K.D.: The design and analysis of block experiments (with discussions). J. Roy. Statist. Soc., B 14 (1952), 45-100.

[11] Wilkinson, G. N.: A general recursive procedure for analysis of variance. Biometrika, 57 (1970), 19-46.

[12] Yamamoto, S. and Fujikoshi, Y.: Two-way classification designs with unequal cell frequencies. J. Sci. Hiroshima Univ., Ser. A-I, 32 (1968), 357-370.

Communicated by Ch. Asano

Received March 21, 1984 